\title{
Meteorite
}

\section{Claudio Giovanardi}

PUBBLICATO: 15 DECEMBER 2020

\section{Quesito:}

Alcuni lettori ci pongono domande a proposito del termine meteorite: è maschile o femminile? E qual è il suo plurale?

\section{Meteorite}

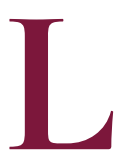

e moderne terminologie scientifiche si sono formate a partire dal XVIII secolo sulla base di un concetto tanto semplice quanto efficace: far corrispondere a fenomeni dello stesso tipo il medesimo elemento linguistico, in modo da creare delle serie tassonomiche potenzialmente infinite dal significato trasparente e univoco. Un passaggio fondamentale, in tale direzione, fu quello di attribuire un significato specifico a determinati affissi, in particolare suffissi, attraverso i quali indicare in modo economico e universalmente condiviso un determinato stato o processo. È il caso, appunto, del suffisso -ite, di origine greca, che viene usato in diversi ambiti scientifici con significati diversi. In medicina il suffisso -ite indica un processo infiammatorio acuto dell'organo cui si riferisce in termini come bronchite, epatite, nefrite, polmonite. Se facciamo attenzione agli esempi appena citati, noteremo un'importante caratteristica della loro formazione: il suffisso -ite può unirsi tanto a parole vere e proprie (bronchi, polmoni), quanto a prefissoidi o confissi (epat(o)-, nefr(o)-), anch'essi di origine greca, i quali sono provvisti di un significato (rispettivamente 'fegato' e 'rene'), ma non hanno autonomia lessicale: non posso infatti dire "mi fa male l'epato, ma dovrò dire mi fa male il fegato.

Il suffisso -ite, oltre che in medicina, è utilizzato anche in altre terminologie scientifiche, tra le quali quella della mineralogia, la scienza che studia la forma, la struttura e le proprietà dei minerali. A tale disciplina, ma all'interno della scienza astronomica, si richiama il termine meteorite, per il quale riportiamo la definizione del GDLI: "Ciascuno dei corpi solidi, di forma tondeggiante o irregolare e di dimensioni variabili da frazioni di millimetri fino a centinaia di metri, di provenienza extraterrestre, vaganti nello spazio, che possono talvolta entrare nel campo d'attrazione della Terra: durante l'attraversamento dell'atmosfera, per attrito con l'aria, si rendono incandescenti, mentre il loro interno può conservare temperature bassissime, e in prossimità o a contatto del suolo, date le tensioni interne, esplodono". Il vocabolo è attestato lessicograficamente per la prima volta nel TommaseoBellini (i869) ed è formato da meteora con il predetto suffisso -ite. A sua volta meteora è una voce dotta che viene dal greco antico ta metéôra 'le cose che stanno in alto' e indica genericamente un fenomeno atmosferico, tant'è vero che la meteorologia (e non “metereologia, come spesso si sente dire) è la scienza che studia i fenomeni atmosferici.

E chiudiamo con i quesiti morfologici. Generalmente i sostantivi in -ite sono femminili (la pirite, la stalattite), ma per meteorite i dizionari indicano entrambi i generi. È probabile che l'uso al maschile sia dovuto all'attrazione di sinonimi quali corpo o aggregato celeste. Quanto al plurale, la forma corretta è, in ogni caso, meteoriti. 


\section{Cita come:}

Claudio Giovanardi, Meteorite , "Italiano digitale", 2020, XV, 2020/4 (ottobre-dicembre) DOI: $10.35948 / 2532-9006 / 2020.5436$

Copyright 2020 Accademia della Crusca

Pubblicato con licenza creative commons CC BY-NC-ND 